\title{
Efeitos das células-tronco mesenquimais no tratamento da osteoartrite de joelho: um relato de caso no Sistema Único de Saúde do Brasil
}

\section{Effects of Adipose-derived Stem Cells in the Treatment of Knee Osteoarthritis: A Case Report in Brazil's Unified Health System}

\author{
Laynna de Carvalho Schweich-Adami,2(1) Roberto Antoniolli da Silva ${ }^{30}$ Adrivanio Baranoski ${ }^{10}$ \\ Candida Aparecida Leite Kassuya ${ }^{4(0)}$ Andréia Conceição Milan Brochado Antoniolli-Silva ${ }^{1,2}$ () \\ Rodrigo Juliano Oliveira ${ }^{1,2,5}$
}

\footnotetext{
${ }^{1}$ Centro de Estudos em Células Tronco, Terapia Celular e Genética Toxicológica - CeTroGen, Hospital Universitário Maria Aparecida Pedrossian - HUMAP/EBSERH, Universidade Federal de Mato Grosso do Sul - UFMS, Campo Grande, Mato Grosso do Sul, MS, Brasil

2 Programa de Pós-graduação em Saúde e Desenvolvimento da Região Centro-Oeste, Faculdade de Medicina Dr. Hélio Mandetta - Famed, Universidade Federal de Mato Grosso do Sul - UFMS, Campo Grande, Mato Grosso do Sul, MS, Brasil

${ }^{3}$ Ambulatório de Ortopedia e Traumatologia, Hospital Universitário Maria Aparecida Pedrossian - HUMAP/EBSERH, Universidade Federal de Mato Grosso do Sul - UFMS, Campo Grande, Mato Grosso do Sul, MS, Brasil
}

\author{
Endereço para correspondência Rodrigo Juliano Oliveira, PhD, \\ Faculdade de Medicina, Universidade Federal de Mato Grosso do Sul, \\ Cidade Universitária s/n, Campo Grande, Mato Grosso do Sul, MS, \\ 79070-900, Brasil (e-mail: rodrigo.oliveira@ufms.br). \\ ${ }^{4}$ Faculdade de Ciências da Saúde - FCS, Universidade Federal da \\ Grande Dourados - UFGD, Dourados, Mato Grosso do Sul, MS, Brasil \\ 5 Programa de Pós-Graduação em Genética e Biologia Molecular, \\ Centro de Ciências Biológicas - CCB, Universidade Estadual de \\ Londrina - UEL, Londrina , Paraná, PR, Brasil
}

Rev Bras Ortop

\begin{abstract}
Resumo
Palavras-chave

- terapia celular

- dor articular

- inflamação

- Sistema Único de Saúde

- ortopedia

- medicina regenerativa

A osteoartrite $(\mathrm{OA})$ pode deixar o indivíduo incapacitado para realizar suas atividades da vida diária devido ao quadro álgico. Essa é uma importante questão de saúde pública que se agrava no mundo inteiro e no Brasil, uma vez que a população passa pelo processo de envelhecimento, e isso causa um aumento nos gastos públicos com o acompanhamento e manutenção dos tratamentos que podem perdurar por anos e mesmo assim não serem resolutivos. Assim, torna-se necessária a busca por terapias inovadoras e eficazes que possam reduzir esses custos. Nesse contexto, o presente estudo relata a primeira aplicação de terapia celular com células-tronco mesenquimais do tecido adiposo no tratamento de OA refratária ao tratamento conservador realizada no Sistema Único de Saúde (SUS). Na avaliação, foram usados os instrumentos Escala Visual Analógica (EVA), os questionários de qualidade de vida Short Form Health Survey (SF-36) e Western Ontario and McMaster Universities (WOMAC), específicos para avaliação da OA, e fez-se uma análise do líquido sinovial (citocinas inflamatórias). A terapia celular melhorou as pontuações no WOMAC, SF-36, e EVA, e reduziu o processo
\end{abstract}

recebido

29 de Outubro de 2020

aceito

11 de Fevereiro de 2021
DOI https://doi.org/

10.1055/s-0041-1733797. ISSN 0102-3616. (c) 2021. Sociedade Brasileira de Ortopedia e Traumatologia. All rights reserved.

This is an open access article published by Thieme under the terms of the Creative Commons Attribution-NonDerivative-NonCommercial-License, permitting copying and reproduction so long as the original work is given appropriate credit. Contents may not be used for commercial purposes, or adapted, remixed, transformed or built upon. (https://creativecommons.org/ licenses/by-nc-nd/4.0/)

Thieme Revinter Publicações Ltda., Rua do Matoso 170, Rio de Janeiro, RJ, CEP 20270-135, Brazil 
inflamatório. Observou-se redução de $0,73 \times$ do TNF, de $0,71 \times$ da IL-1b, de $0,68 \times$ da IL-8, e de $0,70 \times$ da IL-10. Já para a IL-6, observou-se aumento de 1,48 $\times$. Portanto, considera-se este tipo de terapia celular promissora no auxílio do manejo desta doença, pois melhorou o quadro álgico do paciente, reduziu os marcadores inflamatórios, e possibilitou o retorno às atividades da vida diária, o que resultou em uma melhora de sua qualidade de vida.

Abstract

\section{Keywords}

- cell therapy

- joint pain

- inflammation

- Unified Health System

- orthopedics

- regenerative medicine
Osteoarthritis $(\mathrm{OA})$ can incapacitate the individual to perform their activities of daily living due to pain. This is an important public health issue that worsens worldwide and in Brazil, since the population goes through an aging process, and has caused increased public spending on the monitoring and maintenance of treatments that can last for years and still not be resolutive. Thus, the search for innovative and effective therapies that can reduce costs becomes necessary. In this context, the present study reports the first application of cell therapy with adipose-derived stem cells in the treatment of cases of $\mathrm{OA}$ that are refractory to the conservative treatment, performed in the Brazilian Unified Health System (Sistema Único de Saúde, SUS). The evaluation was performed with the application of the Visual Analog Scale (VAS), the Short Form Health Survey (SF36) and the Western Ontario and McMaster Universities (WOMAC), specifics for OA evaluation, and also an analysis of the synovial fluid (inflammatory cytokines). The cell therapy improved the scores on the WOMAC, SF-36 and EVA, and reduced the inflammatory process. We observed a reduction of $0.73 \times$ in the TNF, of $0,71 \times$ in IL-1b, of $0.68 \times$ in IL-8, and of $0,70 \times$ in IL-10. For IL-6, an increase of $1,48 \times$ was observed. Therefore, this cell therapy can be considered promising in aiding the management of this disease, since it improved the patient's pain, reduced inflammatory markers, and enabled the return to activities of daily living, which resulted in an improvement in their quality of life.

\section{Introdução}

As células-tronco mesenquimais (CTMs) do tecido adiposo apresentam resultados interessantes no tratamento de osteoartrite (OA). ${ }^{1}$ Os nossos resultados pré-clínicos indicaram que as CTMs, por efeitos parácrinos e de diferenciação celular, podem levar à melhoria do reparo e da regeneração da cartilagem. ${ }^{2}$ Estes dados foram importantes para iniciarmos um estudo clínico que utilizará as CTMs no tratamento da OA em humanos, e implantar e implementar terapias inovadoras no Centro de Processamento Celular, em parceria com o Serviço de Ortopedia, do Hospital Universitário Maria Aparecida Pedrossian (HUMAP/EBSERH), em Campo Grande, Mato Grasso do Sul (MS), Brasil.

O presente estudo relata os resultados da primeira terapia celular com CTMs do tecido adiposo em um paciente do Sistema Único de Saúde (SUS) no Brasil.

\section{Relato de caso}

Paciente do sexo masculino, com 66 anos, $93 \mathrm{Kg}$ e 1,75 m de altura, com diagnóstico de rotura de menisco medial no joelho direito com OA grau III (na classificação de Kellgren e Lawrence pela análise radiográfica), e prótese total no joelho esquerdo. Na avaliação clínica, o paciente relatou dor intermitente no joelho direito, e apresentou crepitação articular durante a movimentação, aumento da circunferência do joelho, e diminuição da amplitude de movimento (ADM), sendo somente capaz de fletir a perna até $95^{\circ}$ sem doer. Durante a entrevista, foram aplicados a Escala Visual Analógica (EVA), e os questionários de qualidade de vida Short Form Health Survey (SF-36) e Western Ontario and McMaster Universities (WOMAC), este específico para OA. O paciente foi então esclarecido sobre o tratamento com CTMs, e assinou o termo de consentimento livro e esclarecido para a utilização da terapia celular.

Na semana seguinte, a cirurgia de videoartroscopia foi realizada conforme a rotina do Serviço de Ortopedia do Hospital. Foi realizada a limpeza da articulação e debridamento leve da cartilagem afetada (- Figura 1C-D). Após uma semana, o paciente retornou ao hospital para a coleta de tecido adiposo, realizada por lipoaspiração. ${ }^{3}$

O processamento do lipoaspirado, extração, cultivo,caracterização (imunofenotipagem: [cluster of differentiation, $C D$, em inglês] CD105, CD90, CD34 e CD133), e diferenciação celular (adipogênica, condrogênica e osteogênica) das CTMs foram realizados segundo Schweich et al. ${ }^{4}$ (2017). O cultivo das CTMs para o transplante ocorreu por 25 dias ( $3^{\mathrm{a}}$ passagem) até atingir a quantidade necessária (-Figura 2). Foi realizado teste bacteriológico antes da terapia celular. Depois, o paciente 


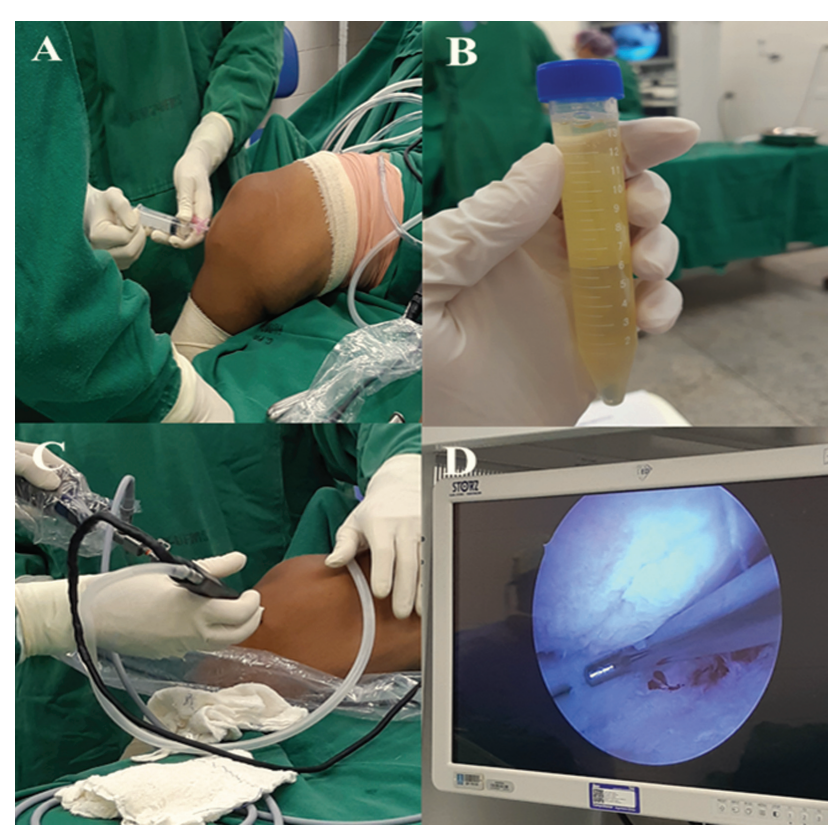

Fig. 1 Procedimento cirúrgico de videoartroscopia. (A) Artrocentese. (B) Líquido sinovial coletado. (C) Posicionamento dos instrumentos cirúrgicos e locais de entrada. (D) Visualização da cartilagem articular para limpeza de vestígios ou áreas sem nivelamento.

retornou e recebeu uma injeção intra-articular contendo $1 \times 10^{7}$ de CTMs homogeneizadas em $3 \mathrm{ml}$ de solução fisiológica (-Figura 2). Aplicou-se uma bandagem em volta do joelho tratado para evitar a flexão do membro nas primeiras 12 horas.

A coleta do líquido sinovial aconteceu em dois momentos: no centro cirúrgico antes da videoartroscopia (- Figura 1A-B), e após seis meses da terapia celular. Para a análise do processo inflamatório, utilizou-se o CBA Human Inflammatory Cytokines KIT (BD Biosciences, Franklin Lakes, NJ, EUA), conforme instruções do fabricante, por citometria de fluxo (Cytoflex, Beckman Coulter, Inc., Brea, CA, EUA).

Em relação aos resultados da terapia celular, observamos que, na avaliação dos domínios do questionário SF-36, a

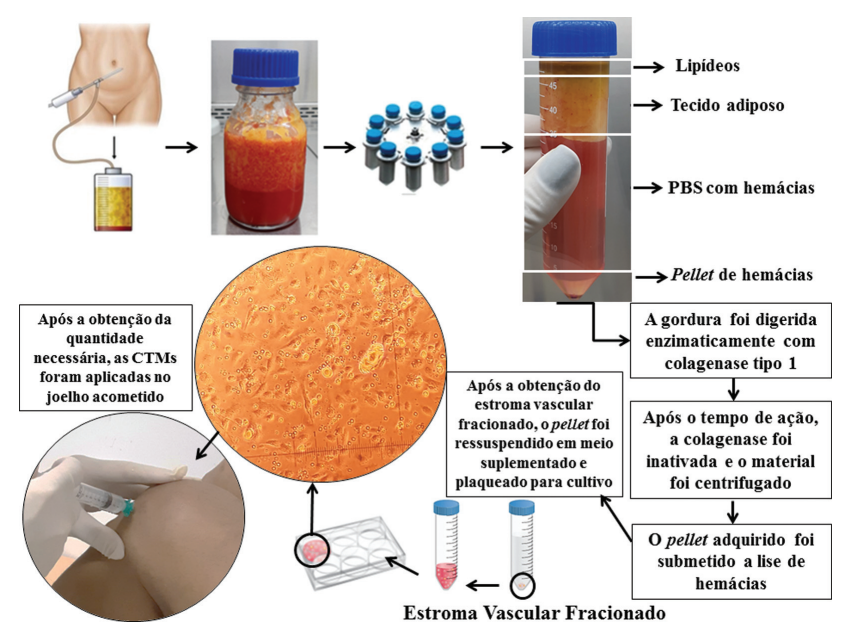

Fig. 2 Fluxograma de procedimentos de obtenção e processamento do lipoaspirado, cultivo das células-tronco mesenquimais, e aplicação no joelho com osteoartrite.

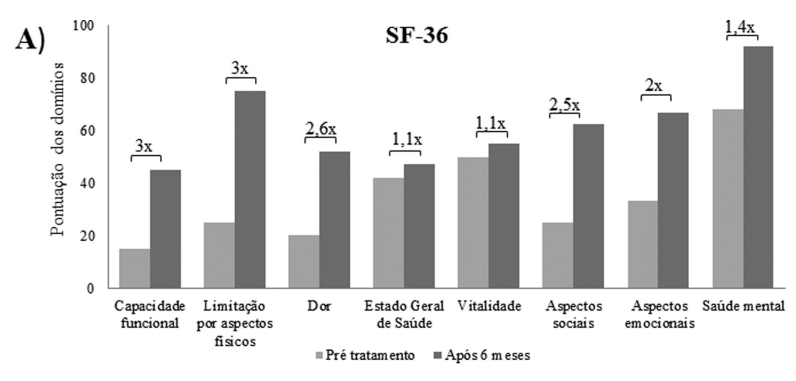

B)
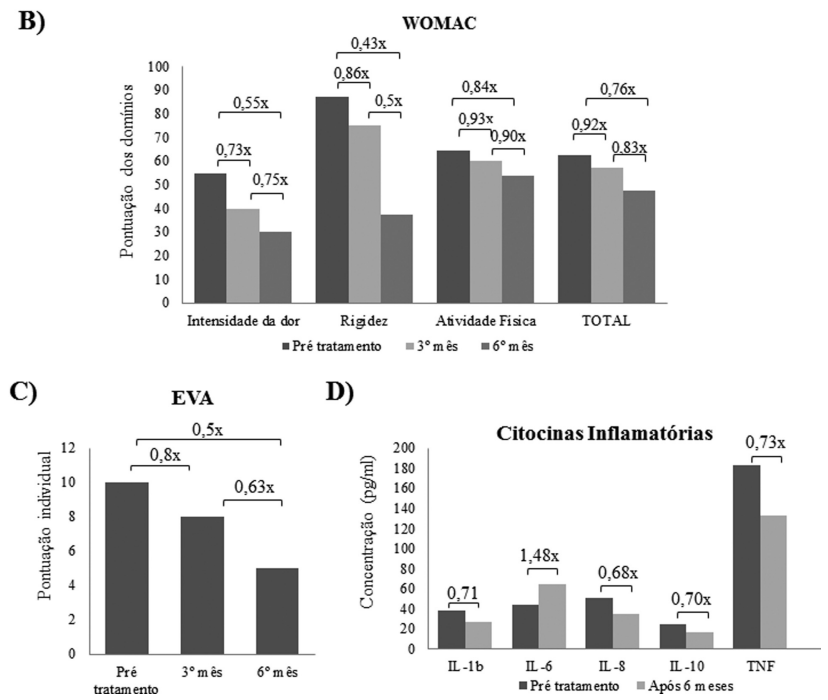

Fig. 3 Valores adquiridos após avaliações propostas no período pré e pós tratamento com CTMs. (A) Pontuação do questionário SF-36 aplicados antes da intervenção e após 6 meses. (B) Pontuação do questionário WOMAC antes da intervenção, no $3^{\circ}$ mês e no $6^{\circ}$ mês. (C) Classificação de EVA realizada antes da intervenção, no $3^{\circ}$ mês e no $6^{\circ}$ mês. (D) Quantificação das citocinas inflamatórias antes da intervenção e no $6^{\circ}$ mês.

capacidade funcional e a limitação por aspectos físicos melhoraram em $3 \times$, a dor em $2,6 \times$, os aspectos sociais em $2,5 \times$, os aspectos emocionais em $2 \times$, a saúde mental em $1,4 \times$, e 0 estado geral de saúde e a vitalidade em 1,1 $\times$ (-Figura 3A).

O questionário WOMAC indicou redução da pontuação dos domínios tanto para a avaliação de três meses quanto para a de seis meses após a terapia celular. Na segunda avaliação, em relação ao quadro inicial, a redução foi de $0,55 \times$ para intensidade da dor, $0,43 \times$ para rigidez, $0,84 \times$ para a atividade física, e $0,76 \times$ na pontuação total ( - Figura 3B).

A pontuação na EVA demonstrou redução de $0,8 \times$ e 0,63 × para primeira e segunda avaliações, respectivamente (-Figura 3C).

A avaliação macroscópica do líquido sinovial demonstrou melhora da viscosidade, redução da opacidade, e maior homogeneidade. A avaliação de citocinas inflamatórias demonstrou redução de $0,73 \times$ do TNF, $0,71 \times$ da IL- 1 b, de $0,68 \times$ da IL-8, e de $0,70 \times$ da IL-10. Já para IL-6, observou-se aumento de $1,48 \times(-$ Figura 3D $)$.

\section{Discussão}

O modo de ação das CTMs no tratamento da OA acontece por meio de três efeitos biológicos diferentes: diferenciação 
celular, modulação inflamatória (efeito parácrino), e mediação de condroprotetores. ${ }^{5}$ Neste relato, os resultados no SF36, no WOMAC e na EVA demonstram que a terapia celular pode melhorar o quadro do paciente com OA, pois melhora a funcionalidade do membro afetado e do paciente como um todo, o que reflete em retorno às atividades da vida diária e melhoria na qualidade de vida global, esses resultados corroboram com a literatura atual. 6,7

Na patogênese da $\mathrm{OA}$, destacam-se a predominância das citocinas IL-6, IL-1b e TNF. Essas citocinas têm a capacidade de ativar múltiplas vias inflamatórias, podendo aumentar a gravidade da doença, o inchaço das articulações, e a destruição da cartilagem. ${ }^{8}$ Neste relato de caso, observou-se também um decréscimo nos níveis de IL-1b, IL-8, IL-10, e principalmente do TNF. Com a redução destas citocinas, pode-se sugerir que houve uma diminuição do processo inflamatório local, o que auxiliou na melhora do quadro degenerativo desta articulação. Porém com a diminuição, em especifico, da IL-10, que é uma citocina anti-inflamatória, ${ }^{9}$ observa-se a necessidade de mais estudos que possam descrever/entender como as CTMs modulam o processo inflamatório nessa doença. A única das citocinas que aumentou foi a IL-6, sendo esta relacionada à ativação de genes-alvo envolvidos na diferenciação celular, proliferação e apoptose. ${ }^{8}$ Assim, inferimos esse aumento devido ao debridamento leve realizado durante a cirurgia de videoartroscopia, pois ele estimula a proliferação/diferenciação.

A redução do processo inflamatório, sugerido pela modulação das citocinas, pode explicar a melhora da viscosidade do líquido sinovial, bem como a redução da opacidade, pois o processo inflamatório causa influxo de células para a cavidade articular. Logo, com a redução do número de células, ocorre redução da opacidade. Soma-se a isso, e ao aumento da homogeneidade, a redução de fibrina e os restos de desgaste da cartilagem que também são favorecidos pelo processo inflamatório que foi reduzido. ${ }^{10}$ Esses fatos são importantes, visto que a qualidade do líquido sinovial reflete na qualidade do tecido cartilaginoso articular.

Infere-se que a terapia celular com CTMs, em pacientes com OA refratária ao tratamento conservador, pode ser considerada uma alternativa promissora no auxílio do manejo dessa doença, pois há melhora do quadro álgico e retorno do paciente às suas atividades da vida diária.

\section{Conflito de interesses}

Os autores declaram não haver conflito de interesses.

\section{Agradecimentos}

À Dra. Tatyanne Ferreira da Silva, pelo procedimento de lipoaspiração. À Fundect, ao CNPq e à Capes, pelo apoio financeiro.

\section{Referências}

1 Biazzo A, D’Ambrosi R, Masia F, Izzo V, Verde F. Autologous adipose stem cell therapy for knee osteoarthritis: where are we now? Phys Sportsmed 2020;48(04):392-399

2 Hermeto LC, DeRossi R, Oliveira RJ, et al. Effects of intra-articular injection of mesenchymal stem cells associated with platelet-rich plasma in a rabbit model of osteoarthritis. Genet Mol Res 2016;15 (03):10.4238/gmr.15038569

3 Pesarini JR, Oliveira RJ, Pessatto LR, et al. Vitamin D: Correlation with biochemical and body composition changes in a southern Brazilian population and induction of cytotoxicity in mesenchymal stem cells derived from human adipose tissue. Biomed Pharmacother 2017;91:861-871

4 Schweich LC, Oliveira EJT, Pesarini JR, et al. All-trans retinoic acid induces mitochondria-mediated apoptosis of human adiposederived stem cells and affects the balance of the adipogenic differentiation. Biomed Pharmacother 2017;96:1267-1274

5 Lee WS, Kim HJ, Kim KI, Kim GB, Jin W. Intra-articular injection of autologous adipose tissue-derived mesenchymal stem cells for the treatment of knee osteoarthritis: a phase IIb, randomized, placebo-controlled clinical trial. Stem Cells Transl Med 2019;8 (06):504-511

6 Lu L, Dai C, Zhang Z, et al. Treatment of knee osteoarthritis with intra-articular injection of autologous adipose-derived mesenchymal progenitor cells: a prospective, randomized, doubleblind, active-controlled, phase IIb clinical trial. Stem Cell Res Ther 2019; 10(1-10):143

7 Pers YM, Rackwitz L, Ferreira R, et al; ADIPOA Consortium. Adipose Mesenchymal Stromal Cell-Based Therapy for Severe Osteoarthritis of the Knee: A Phase I Dose-Escalation Trial. Stem Cells Transl Med 2016;5(07):847-856

8 Saraiva M, O'Garra A. The regulation of IL-10 production by immune cells. Nat Rev Immunol 2010;10(03):170-181

9 Wang T, He C. Pro-inflammatory cytokines: The link between obesity and osteoarthritis. Cytokine Growth Factor Rev 2018; 44:38-50

10 Franco RN, Cintra Neto PF, Pimentel ER, Cohen M, Lima GEG, Mattiello-Rosa SMG. Correlation between inflammatory cells and sulfated glycosaminoglycan concentration in synovial fluid of subjects with secondary knee osteoarthritis. J Rheumatol 2008; 35(06):1096-1101 